\title{
Imported new world cutaneous leishmaniasis in a traveller from Slovakia
}

\author{
Ondriska $\mathrm{F}^{1}$, Bukovinova $\mathrm{P}^{2}$, Votypka $\mathrm{J}^{3}$, Nohynkova $\mathrm{E}^{4}$, Boldis $\mathrm{V}^{1}$ \\ HPL (Ltd) Medical Laboratories, Department of Parasitology, Bratislava, Slovakia. ondriska@hpl.sk
}

\begin{abstract}
We present a case of imported leishmaniasis in a 31-year-old woman from Slovakia, who visited the countries of South America for three months in 2011. On 29 and 31 August 2011, she was probably infected with Leishmania parasites in the jungles of Ecuador. Approximately one week after returning to Slovakia, a small papules appeared on patient's left leg. Another wound was found after two weeks. Both ulcers were enlarging. We proved amastigote forms of Leishmania spp. only in repeated dermal scrapings from the edge of the ulcer by Giemsa staining after negative results from examination of a wound scrape and biopsy specimen. We identified the species Leishmania (Viannia) panamensis as a causative agent by using the polymerase chain reaction (PCR) method and subsequent sequencing of the ITS region. Closure of wounds and scab formation were observed after 20 days of treatment with sodium stibogluconate. In the control microscopic examination after the end of the treatment, parasites were not present, and the PCR confirmed the negative result (Fig. 2, Ref. 31). Text in PDF www.elis.sk.

Key words: cutaneous leishmaniasis, Leishmania (Viannia) panamensis, South America, travel.
\end{abstract}

\section{Introduction}

Leishmaniasis is a vector-born parasitic disease caused by several species of protozoan parasites of the genus Leishmania and transmitted by the bite of phlebotomine sandflies. It is endemic in 88 countries around the world, ranging from the tropics to the subtropics, including southern Europe. The number of human infections has reached about 12 million, with an annual incidence of two million new cases, and an additional 350 million people are estimated to be at risk (1). There are two main clinical forms of leishmaniasis: (i) visceral (VL) and (ii) cutaneous (CL), which represents about $2 / 3$ of all clinical cases. Cutaneous leishmaniasis in the New World is caused by protozoans of the genus Leishmania, which has been divided into two subgenera: Leishmania and Viannia. The reservoir of infection is a wide range of vertebrates, including humans $(2,3)$. The most common clinical form of $\mathrm{CL}$ presents as single or multiple localised skin lesions. At the bite site, on the face, back, or extremities, CL starts as a painless, often itchy papule that ulcerates to a typical round-to-oval lesion with a necrotic and exudative centre surrounded by a raised margin. The

${ }^{1}$ HPL (Ltd) Medical Laboratories, Department of Parasitology, Bratislava, Slovakia, ${ }^{2}$ Department of Infectology and Geographical Medicine, Derer's Hospital, Bratislava, Slovakia, ${ }^{3}$ Department of Parasitology, Charles University in Prague, Faculty of Science, Prague, Czech Republic, and ${ }^{4}$ Department of Tropical Medicine, 1(st) Faculty of Medicine, Charles University in Prague and Hospital Bulovka, Prague, Czech Republic

Address for correspondence: F. Ondriska, $\mathrm{Dr}, \mathrm{PhD}$, Department of Parasitology, HPL (Ltd) Medical Laboratories, Istrijska 20, SK-841 07 Bratislava, Slovakia.

Phone: +421.2 .69307130$ lesions are usually painless but can be painful, especially if ulcerative lesions become infected with bacteria or if the lesions are near a joint. Sometimes lymphadenopathy is noticed first, before skin lesions develop. The healing process typically results in atrophic scarring $(4,5)$. Third, less common form of the disease, mucocutaneous leishmaniasis (MCL) develops in $1-10 \%$ of patients with localised CL caused by Leishmania or Viannia subgenera, mainly in Latin America $(6,7)$. Therefore, the causative agent of CL imported from Latin America should be identified before treatment. Diagnosis is confirmed by a microscopic and cultivation evidence of the parasite from biopsies of skin lesions. Because Leishmania species are indistinguishable microscopically, differentiation is based on molecular, biological (polymerase chain reaction (PCR)) and biochemical techniques $(8,9,10,11)$.

Autochthonous leishmaniasis is not found in countries located in the temperate climate zone, including Slovakia. To date, two cases of imported leishmaniasis in travellers returning from endemic areas have been documented in Slovakia $(12,13,14)$. We report a case of imported CL in a young traveller returning from Latin America.

\section{Case report}

A 31-year-old woman travelled through the countries of South America between 2 August and 14 October 2011. She spent 2 days in Bolivia, 10 days rafting in Peru, and one and a half months in Ecuador (from 16 August to 8 October 2011). During this period, she visited the jungles of Santo Domingo in the community Tsachilas for 3 days (from 29 to 31 August 2011). After this trip, she travelled back to Peru and Bolivia and returned to Slovakia. She 

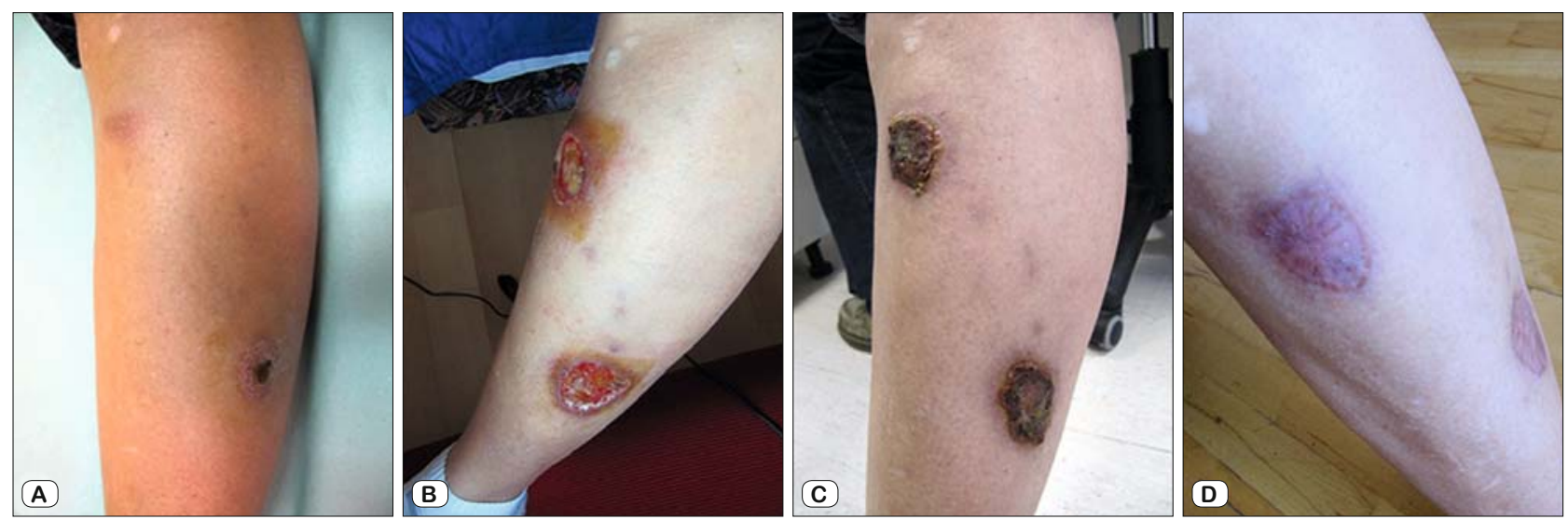

Fig. 1. A) - The patient's left leg about a week after the return to Slovakia (on 21 October 2011). B) - Ulcers on 21 December 2011 (the day when the biological specimens were obtained by scraping the edge of the lesion). C) - The patient's left leg one week after the treatment (on 14 March 2012). CL ulcers were significantly improved, and closure of wounds and scab formation were observed. D) - The scars one year after the treatment.

had no health problems during the stay in South America. About a week after her return to Slovakia (on 21 October 2011), an ulcer of $2 \mathrm{~cm}$ in diameter developed from a small papule on her shin of the left leg in the distal calf. The ulceration was characterised by an increasing size, an intermittent yellowish discharge, and small crust on the surface. A new wound appeared at approximately 7 $\mathrm{cm}$ above the primary lesion, towards the knee (Fig. 1a). She had a normal body temperature and the lesions were not itchy. On 16 November 2011, a local physician obtained biological specimens by scraping the edge of the lesions. No Leishmania parasites were found by microscopic examination of Giemsa-stained slides. The patient's serum did not show any haemagglutination activity in an indirect haemagglutination test for the detection of antibodies. Treatment with clindamycin was initiated due to a positive culture result for Staphylococcus aureus. Slightly elevated C-reactive protein (CRP; $8.58 \mathrm{mg} / \mathrm{L}$; normal range $0-5 \mathrm{mg} / \mathrm{L}$ ) and lipase levels (1.61 $\mu \mathrm{kat} / \mathrm{L}$; reference range $0.22-1.00 \mu \mathrm{kat} / \mathrm{L})$ were detected by laboratory methods used in haematology and biochemistry. Complete blood count was normal except for slightly elevated monocytes. On 29 November 2011, a part of the wound (below the knee) was surgically excised for histological examination. The epidermis showed irregular acanthosis. Dilated capillaries and dense lymphocytic infiltrate with an admixture of histiocytes were found in the upper and middle epidermis. The lower dermis and subcutis were not present. Infections of macrophages by amastigote forms of Leishmania spp. were not found in this sample. The dimensions of the lesions further increased. A pink-red painless plaque (5 $\mathrm{cm}$ long $x 1 \mathrm{~cm}$ wide) appeared in the distal $1 / 3$ of the posterior thigh. Swollen inguinal lymph nodes were not seen. The patient was transferred to our laboratory (HPL Ltd. Slovakia) due to persistent clinical suspicion for leishmaniasis. On 21 December 2011, we obtained biological specimens by scraping the edge of the lesion (Fig. 1b), and showed amastigote forms of Leishmania spp. by Giemsa staining (Fig. 2a).

The titres of haemagglutination activity of the patient's serum were borderline (1:16). A DNA sample from the patient's lesion was analysed by Leishmania-specific PCR amplification using the primer pair of L5.8S and LITSR (15), which amplifies approximately 300-bp amplicon containing a partial sequence of the $18 \mathrm{~S}$ ribosomal RNA gene, complete sequences of internal transcribed spacer 1 (ITS1) and a partial sequence of the $5.8 \mathrm{~S}$ ribosomal RNA gene (Fig. 2b). The final PCR product was gel-purified and sequenced using the BigDye Terminator version 3.1 Cycle Sequencing kit (Applied Biosystems, Foster City, CA, USA) on
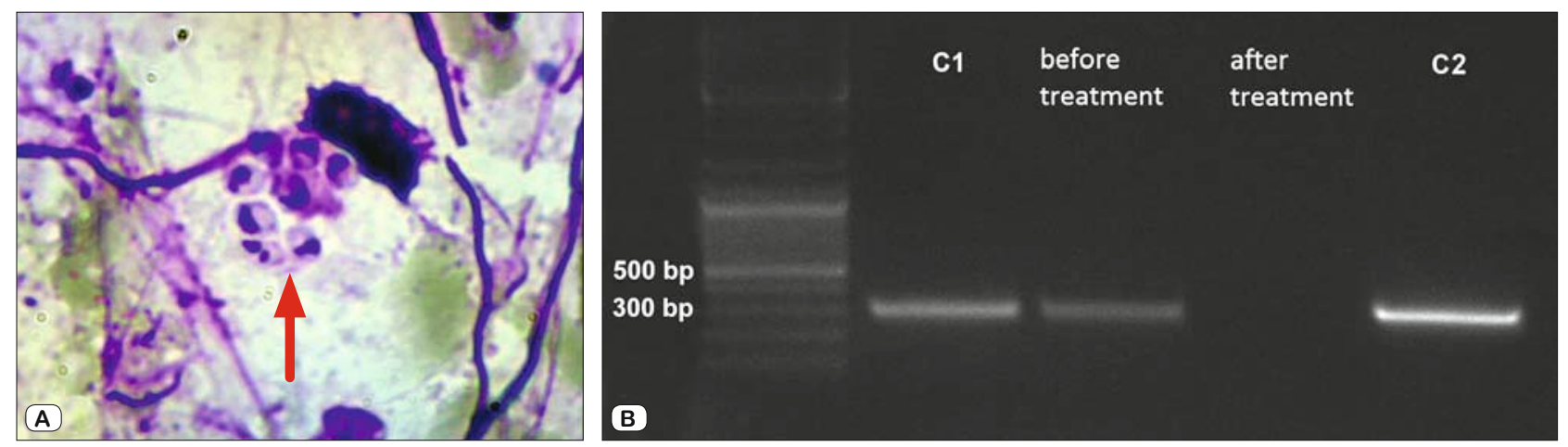

Fig. 2. A) - Microscopic image of amastigote forms of Leishmania spp. stained by Giemsa. B) - PCR amplification of Leishmania DNA isolated from the patient: $\mathrm{P}$ - patient's sample before/after treatment, control mouse tissue infected by $L$. major (C1) and $L$. (V.) brasiliensis (C2). 
the ABI PRISM 3130 DNA automated sequencer (Applied Biosystems Foster City, CA, USA). Sequencing data were analysed using DNAStar software (Madison, WI, USA) and compared with entries retrieved from GenBank, resulting in the identification of Leishmania (Viannia) panamensis. The recommended treatment for CL was sodium stibogluconate (SSG; Pentostam), based on the mentioned results. The SSG was brought from the United Kingdom because this product was not registered in the Slovakia. On 20 February 2012, parenteral treatment with SSG at a dosage of $20 \mathrm{mg} / \mathrm{kg} /$ day for 20 days was started. Electrocardiographic (ECG) monitoring was carried out during therapy and did not show any deviations. An ultrasonographic scan did not show any pathological changes in parenchymal organs of the abdominal cavity. In repeated control examinations, the blood count data and biochemical markers did not show any deviations from mean values. CL ulcers were significantly improved, and closure of wounds and scab formation were observed after treatment (Fig. 1c). Skin lesions were treated with Betadine also. The patient had no complications after completing treatment, and was discharged into out patient care. On 1 April 2012 (3 weeks after treatment), the titres of haemagglutination activity of the patient's serum were 1:64 in the control serological test. Parasite DNA was not detected by PCR in the specimens scraped from healing wounds (Fig. 2b). Two months after treatment, crusted lesions were no longer present. As typical, the healing process resulted in atrophic scarring that was visible 1 year after treatment (Fig. 1d). On 17 May 2012, the titres of the patient's serum were reduced to borderline values (1:8) by haemagglutination assay in the follow-up examination, and on 17 December 2012, her serum was negative for haemagglutination antibodies.

\section{Discussion}

The increasing trends of global travel, especially to tropical and subtropical countries including South America, pose a higher risk of acquiring CL. An increasing number of CL cases in European travellers was demonstrated in some epidemiological studies. For example, during the last decade, an increasing number of imported CL cases was observed in English and Dutch patients who visited the aforementioned countries $(16,17)$. The highest numbers of CL cases were recorded during a stay in rural and jungle areas. The 31-year-old Slovak traveller reported here was infected while visiting South America. She was probably infected with Leishmania by a sand fly bite in the jungles of Santo Domingo (Ecuador). Native people informed the patient about the presence of leishmaniasis and its vector, phlebotomine sandflies of the genus Lutzomyia, in Ecuador. CL and MCL constitute a serious public health problem in Ecuador, since the diseases are endemic in 17 of 20 provinces (18). The complex Leishmania (Viannia) panamensis/guyanensis is the predominant aetiologic agent of CL and MCL in Ecuador, and was the agent identified in our patient. In addition to this complex, a further five species of Leishmania have been recorded in Ecuador $(1,11,19,20)$.

The diagnosis of $\mathrm{CL}$ is difficult due to the wide range of cutaneous manifestations. Differential diagnosis is necessary to dis- tinguish CL from cutaneous malignancies, bacterial and fungal skin lesions, leprosy, tuberculosis, eczema, and myiasis, which could have similar symptoms $(21,22)$. One should consider CL with moist non-healing papular or ulcerative progressive lesions on the skin in people residing in endemic areas. We observed these symptoms in our patient; therefore, we preferentially focused on the diagnosis of leishmaniasis. Although South American CL may be often accompanied by swollen lymph glands or secondary pustules on mucous membranes that should also be thoroughly investigated (16), we did not observe these symptoms in our patient. The patient did not suffer from fever and other examination results were normal except for a slightly elevated CRP.

The standard and still the most commonly used diagnostic method is microscopic examination of lesion material obtained by biopsy or scraping after Giemsa staining or after histological processing $(4,6)$. As we confirmed in our patient, the success of diagnosis depends on correct sampling from the proper place on the lesion (23). Histological examination of the sample from excision was not successful; we did not identify Leishmania parasites microscopically even in the first specimens prepared from the ulcer. The amount of Gram + cocci (probably secondary staphylococcal contamination) and leukocytes (PMN) in these preparations also indicated that the material was taken from an opened festering ulcer area where it was not possible to capture Leishmania, because they are destroyed by the immune response. We were able to detect Leishmania only in the repeated scalpel scrapes of the edge that surrounded the central necrotic ulcer area. However, amastigotes were found in such samples only occasionally. This confirmed that in CL, parasites can only be detected in the area of inflammatory skin lesion outside the necrotic tissue, and that the amount of Leishmania can be low and, therefore, requires careful microscopic examination. We carried out identification of the species of parasites isolated from the patient and also used PCR for the verification of the presence of parasites after the treatment. The PCR method together with sequencing can be used for the determination of a species-specific diagnosis because of the morphological similarity of Leishmania species $(11,17)$, and eventually for monitoring treatment success (4).

The aim of treatment of CL is to accelerate the healing of skin lesions and, in the case of New World CL caused by the Viannia subgenus, to prevent the invasion of the parasite into mucous membranes, usually of the oral or nasal cavities $(5,24)$. For the treatment of New World CL, preparations with pentavalent antimony, Glucantime (which contains meglumine antimoniate), Pentostam (which contains sodium stibogluconate), pentamidine isethionate, or azole preparations, all administered parenterally, can be used $(5,16)$. Miltefosine showed high in vitro activity against Leishmania $(25,26,27)$, a single drug against Leishmania that is administered orally $(28,29)$. For our patient, we chose the overall treatment with sodium stibogluconate that is still one of the medications of choice for CL caused by the species of the $L$. (V.) guyanensis complex $(21,30)$. Despite the described adverse drug reactions (17), the patient tolerated the therapy without any problems. The 20-day treatment was successful, which was confirmed by the negative PCR results and microscopic examination about 
a month after the treatment finished. Regular inspections showed no signs of relapse even after 1 year. In the literature, there are two records of imported leishmaniasis among Slovak citizens. The first case was a CL acquired in Iraq in an adult patient (12). The second case was a VL in a 13-month-old girl who was infected in Italy $(13,14)$. In neither case was identification of the Leishmania species performed, although the VL case may have been caused by Leishmania infantum, because the other species that causes the visceral form of the disease, Leishmania donovani, is not present in Europe (1, 31).

Increasing travel opportunities for our citizens to endemic areas bring a considerable risk of serious infection with a broad spectrum of diseases, including CL. Therefore, it is necessary to extend the diagnosis of skin diseases with leishmaniasis for travellers with positive anamnesis.

\section{References}

1. WHO. Control of leishmaniases: report of a meeting of the WHO expert committee on the control of Leishmaniases. Technical report series 949. Geneva, 22-26 March 2010.

2. Dereure J, Espinel I, Barrera C et al. Leishmaniasis in Ecuador. 4. Natural infestation of the dog by Leishmania panamensis. Ann Soc Belg Med Trop 1994; 74 (1): 29-33.

3. Herwaldt BL. Leishmaniasis. Lancet 1999; 354 (9185): 1191-1199.

4. Reithinger R, Dujardin JC, Louzir H, Pirmez C, Alexander B, Brooder S. Cutaneous leishmaniasis. Lancet Infect Dis 2007; 7: 581-596.

5. David CV, Craft N. Cutaneous and mucocutaneous leishmaniasis. Dermatol Ther 2009; 22 (6): 491-502.

6. Davies CR, Reithinger R, Campbell-Lendrum D, Feliciangeli D, Borges R, Rodriguez N. The epidemiology and control of leishmaniasis in Andean countries. Cad. Saúde Pública, Rio de Janeiro 2000; 16 (4): 925-950.

7. Amato VS, Tuon FF, Bacha HA, Neto VA, Nicodemo AC. Mucosal leishmaniasis. Current scenario and prospects for treatment. Acta Trop 2008; 105 (1): 1-9.

8. Rodrígues N, Guzman B, Rodas A, Takiff H, Bloom BR, Convit J. Diagnosis of cutaneous leishmaniasis and species discrimination of parasites by PCR and hybridization. J Clin Microbiol 1994; 32 (9): 2246-2252.

9. Reithinger R, Dujardin JC. Molecular diagnosis of leishmaniasis: current status and future applications. J Clin Microbiol 2007; 45: 21-25.

10. Malekpour M, Esfandbood M. Cutaneous leishmaniasis. N Engl J Med 2010362 (6): e15.

11. Montalvo Alvarez AM, Nodarse JF, Goodridga IM et al. Differentiation of Leishmania (Viannia) panamensis and Leishmania (V.) guyanensis using BccI for hsp70 PCR-RFLP. Trans R Soc Trop Med Hyg 2010; 104 (5): 364-367.

12. Buchvald J, Polák I. Skin Leishmaniasis in Slovakia. Cesk Dermatol 1982; 58 (2): 53-56 (in Slovak).

13. Lukáč J, Székyová D, Mažáriová O, Čáp J. Visceral form of leishmaniosis in a 13-month child. Čes-slov Pediat 2006; 61 (3): 164-167.
14. Székyová D, Lukáč J, Mažáriová O, Čáp J. „Unexplained fever” of holiday. Pediatr prax 2006; 3: 150-151 (in Slovak).

15. Schönian G, Nasereddin A, Dinse $\mathbf{N}$ et al. PCR diagnosis and characterization of Leishmania in local and imported clinical samples. Diagn Microbiol Infect Dis 2003; 47 (1): 349-358.

16. Lawn SD, Whetham J, Chiodini PL et al. New world mucosal and cutaneous leishmaniasis: an emerging health problem among British travellers. Q J Med 2004; 97: 781-788.

17. Schwartz E, Hatz C, Blum J. New world cutaneous leishmaniasis in travellers. Lancet Infect Dis 2006; 6 (6): 342-349.

18. Bañuls AL, Jonquieres R, Guerrini $F$ et al. Genetic analysis of Leishmania parasites in Ecuador. Are Leishmania (Viannia) panamensis and Leishmania (V.) guyanensis distinct Taxa? Am J Trop Med Hyg 1999; 61 (5): 838-845.

19. Armijos RX, Weigel MM, Izurieta R et al. The epidemiology of cutaneous leishmaniasis in subtropical Ecuador. Trop Med Int Health 1990; 2: $140-152$.

20. Calvopina M, Armijos RX, Hashiguchi Y. Epidemiology of leishmaniasis in Ecuador: current status of knowledge - A review. Mem. Inst. Oswaldo Cruz 2004; (99) 7: 663-672.

21. Markle WH, Makhoul K. Cutaneous leishmaniasis: Recognition and treatment. Am Fam Physician 2004; 69 (6): 1456-1460.

22. Recalcati S, Vezzoli P, Girgenti V, Venegoni L, Veraldi S, Berti E. Cutaneous lymphoid hyperplasia associated with Leishmania panamensis infection. Acta Derm Venerol 2010; 90: 418-419.

23. Ramírez JR, Agudelo S, Muskus $C$ et al. Diagnosis of cutaneous leishmaniasis in colombia: the sampling site within lesions influences the sensitivity of parasitologic diagnosis. J Clin.Microbiol 2000; 38 (10): 3768-3773.

24. Minodier P, Parola P. Cutaneous leishmaniasis treatment. Travel Med Infect Dis 2007; 5: 150-158.

25. Soto J, Arana BA, Toledo J et al. Miltefosine for New World cutaneous leishmaniasis. Clin Infect Dis 2004; 38: 1266-1282.

26. Machado PR, Ampuero J, Guimarães LH et al. Miltefosine in the treatment of cutaneous leishmaniasis caused by Leishmania braziliensis in Brazil: a randomized and controlled trial. PLoS Negl Trop Dis 2010; 214 (12): e912.

27. Machado PR, Penna G. Miltefosine and cutaneous leishmaniasis. Curr Opin Infect Dis 2012; 25 (2): 141-144.

28. Lobovská A, Nohýnková E. New antiparasitic drugs. Cas Lek Cesk, 2003; 142 (3): 177-181 (in Czech).

29. Mihál V, Pospíšilová D, Suková M, Starý J. Visceral leishmaniasis treatment progress Klinická farmakologie a farmacie 2005; 19 (2): 100-105 (in Czech).

30. Blum J, Desjeux P, Schwartz E, Beck B, Hatz C. Treatment of cutaneous leishmaniasis among travellers. J Antimicrob Chemother 2004; 53 (2): $158-166$.

31. Ready PD. Leishmaniasis emergence in Europe. Euro Surveill 2010; 15 (10): $1-11$ 